\title{
Investigation on radiation generated by sub-GeV electrons in ultrashort silicon and germanium bent crystals
}

\author{
L. Bandiera ${ }^{1, a}$, A. Sytov ${ }^{1}$, D. De Salvador ${ }^{2,3}$, A. Mazzolari $^{1}$, E. Bagli ${ }^{1}$, R. Camattari ${ }^{1,4}$, S. Carturan ${ }^{2,3}$, \\ C. Durighello ${ }^{1,2,3}$, G. Germogli ${ }^{1}$, V. Guidi ${ }^{1,4}$, P. Klag ${ }^{5}$, W. Lauth ${ }^{5}$, G. Maggioni ${ }^{2,3}$, V. Mascagna ${ }^{6,7}$, M. Prest ${ }^{6,7}$, \\ M. Romagnoni ${ }^{1,8}$, M. Soldani ${ }^{1,4}$, V. V. Tikhomirov ${ }^{9}$, E. Vallazza ${ }^{6}$ \\ ${ }^{1}$ INFN Section of Ferrara, Via Saragat 1, 44122 Ferrara, Italy \\ 2 INFN Laboratori Nazionali di Legnaro, Viale dell'Università 2, 35020 Legnaro, Italy \\ ${ }^{3}$ Department of Physics, University of Padova, Via Marzolo 8, 35131 Padua, Italy \\ ${ }^{4}$ Department of Physics and Earth Sciences, University of Ferrara, Via Saragat 1, 44122 Ferrara, Italy \\ ${ }^{5}$ Institut für Kernphysik der Universität Mainz, 55099 Mainz, Germany \\ ${ }^{6}$ INFN Section of Milano Bicocca, Piazza della Scienza, 3, 20126 Milan, Italy \\ ${ }^{7}$ Dipartimento di Scienza e Alta Tecnologia (DiSAT), University of Insubria, Via Valleggio 11, 22100 Como, Italy \\ ${ }^{8}$ Department of Physics, University of Milan, Via Giovanni Celoria 16, 20133 Milan, Italy \\ ${ }^{9}$ Institute for Nuclear Problems, Belarusian State University, Bobruiskaya 11, 220030 Minsk, Belarus
}

Received: 4 July 2020 / Accepted: 22 March 2021 / Published online: 2 April 2021

(C) The Author(s) 2021

\begin{abstract}
We report on the measurements of the spectra of gamma radiation generated by $855 \mathrm{MeV}$ electrons in bent silicon and germanium crystals at MAMI (MAinzer MIkrotron). The crystals were $15 \mu \mathrm{m}$ thick along the beam direction to ensure high deflection efficiency. Their (111) crystalline planes were bent by means of a piezo-actuated mechanical holder, which allowed to remotely change the crystal curvature. In such a way it was possible to investigate the radiation emitted under planar channeling and volume reflection as a function of the curvature of the crystalline planes. We showed that, using volume reflection, intense gamma radiation can be produced - with intensity comparable to that obtained in channeling but with higher angular acceptance. We studied the trade-off between radiation intensity and angular acceptance at different values of the crystal curvature. The measurements of radiation spectra have been carried out for the first time in bent germanium crystals. In particular, the intensity of radiation in the germanium crystal is higher than in the silicon one due to the higher atomic number, which is important for the development of the X-ray and gamma radiation sources based on higher- $Z$ deformed crystals, such as crystalline undulators.
\end{abstract}

\section{Introduction}

Since the 1960s, it has been known that electrons and positrons interacting with strong crystalline fields can gener-

a e-mail: bandiera@fe.infn.it (corresponding author) ate radiation which is more intense than that resulting from standard bremsstrahlung [1-3]. Indeed, the electric field of a crystalline plane or axis can be very high, reaching the values of $10^{9}-10^{12} \mathrm{~V} / \mathrm{cm}$. For comparison, such a value cannot be artificially created by magnets or by electrostatic deflectors. The first effect discovered was the coherent bremsstrahlung (CB) [1], which occurs when the momentum transferred by the electron/positron to the medium matches a reciprocal lattice vector. It manifests itself for small incidence angle of the charged particles w.r.t. the crystal planes or axes. On the other hand, if the incidence angle of electron/positron trajectory is lower than the critical value, namely the Lindhard angle $\theta_{L}=\sqrt{2 U_{0} / E}$ [4], $U_{0}$ being the planar/axial potential well depth and $E$ the initial particle energy, the particle is forced to move almost parallel to the planes, oscillating between/around them, depending on the charge sign. This is the channeling effect [4], while the radiation produced during such a motion is called channeling radiation (CR) $[5,6]$. $\mathrm{CR}$ is typically softer but more intense than the CB. However, $\mathrm{CR}$ is limited by a low angular acceptance, i.e. within the Lindhard angle, which decreases with the square root of the particle energy. Another limit of CR is dechanneling $[7,8]$, i.e. the process for which charged particles escape from the channeling condition due to scattering of the particles on nuclei and electrons of the crystal. Since the intensity of CR depends on the number of charged particles fulfilling the channeling condition, the crystal length must be at most comparable with the dechanneling length, namely the typical length after which a channeled particle suffers the 


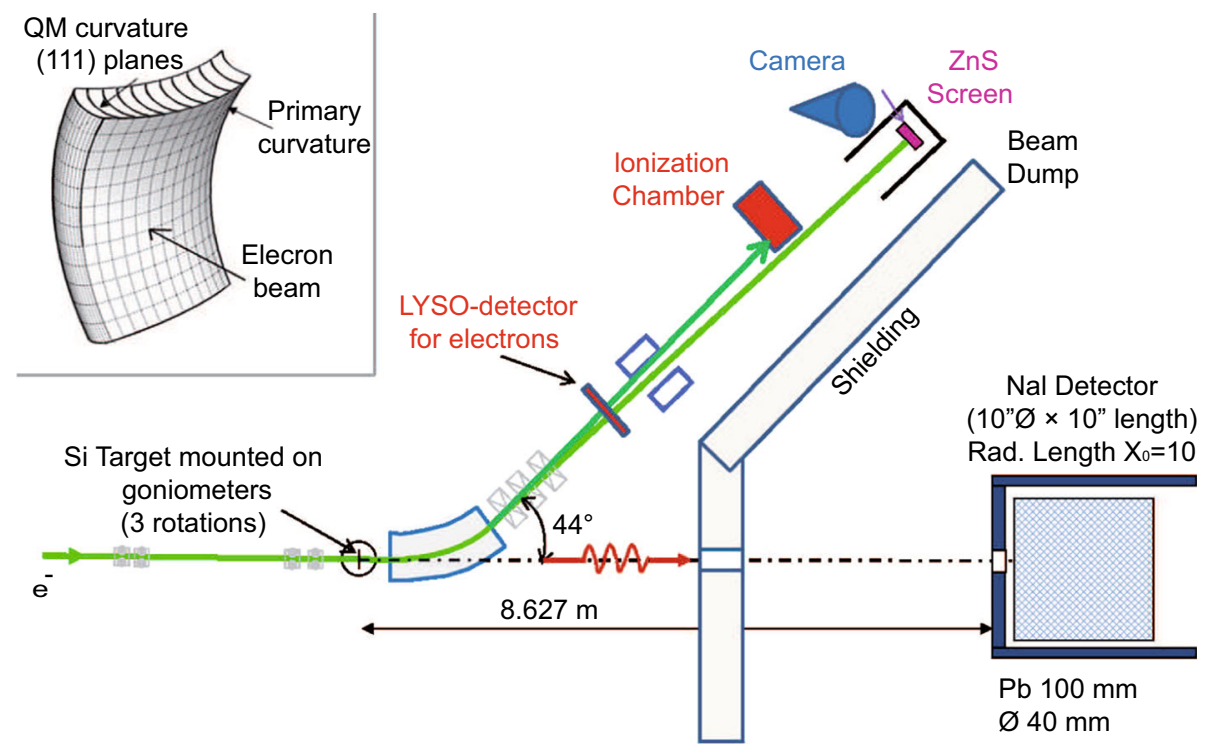

Fig. 1 Scheme of the experimental setup at MAMI B. After the interaction with $\mathrm{Si}$ target mounted on a high precision goniometer, the $855 \mathrm{MeV}$ electron beam is deflected horizontally by a bending magnet. A LYSO detector downstream of the crystal measures the beam distribution after interaction. Then a ionization chamber is positioned after a second dipole-magnet, which deflects particles along the vertical direction. It is employed to detect channeling. Just in front of the beam

dechanneling effect. Soon after its discovery, channeling has been extensively investigated in experiments with $\mathrm{MeV}$ and $\mathrm{GeV}$ electrons and positrons [9-15] and the possibility of constructing intense tunable $\mathrm{X}$ - and $\gamma$-ray sources has been explored.

In the last decade, channeling and the related effects have been investigated in bent crystals, mostly for beam steering applied to collimation and extraction in hadronic accelerators [16-20]. Recently, such investigations were extended to the cases of electrons and positrons [21-29], for which the processes of radiation emission are modified by the presence of the crystal curvature. In fact, a bent crystal possesses a larger variety of effects than a straight one. An effect that occurs only in bent crystals is the so-called volume reflection (VR) [30], in which the particle trajectory is reflected by bent crystal planes if its incidence angle is a little bit higher than the Lindhard angle. Since VR occurs for over-barrier particles, i.e. for not channeled particles, it is not affected by dechanneling. Therefore, VR has a higher deflection efficiency than channeling, while its deflection angle is comparable to $\theta_{L}$. In fact, the VR deflection efficiency is limited by the contribution of volume capture, which consists in the trapping of overbarrier particles in the channeling regime caused by incoherent scattering with lattice atoms while crossing the crystal plane near the reflection point. Nevertheless, the VR deflection efficiency remains higher than for channeling [27,28]. On the other hand, VR is limited by a deflection angle of the dump the beam spot can be monitored with a $\mathrm{ZnS}$ luminescent screen and a CCD camera. The photon beam its a NaI scintillator detector after $8.627 \mathrm{~m}$ from the crystal. The NaI detector is shielded by a $100 \mathrm{~mm}$ thick lead wall with a $40 \mathrm{~mm}$ opening to accept most of the photons. Top-left corner: sketch of the bent crystals. Primary curvature, quasimosaic curvature, and crystallographic orientation of the channeling planes are highlighted

order of $\theta_{L}$, which would make it unsuitable for applications that require larger deflection. Nevertheless, since VR is not affected by dechanneling, thicker crystals can be employed.

VR results in the emission of radiation [31,32], which has a higher angular acceptance w.r.t. CR, being equal to the bending angle of the crystal [24-26], while the radiation intensity is comparable for both the effects. In particular, the radiation accompanying VR could be exploited to develop intense radiation sources with poor beam emittance [26] with the addition of beam deflection, which may find application in the collimation of future linear colliders [33], in the extraction from electron accelerators and for the development of volume-reflecting undulators as proposed in [34]. In fact, another mechanism of radiation generation in a bent crystal is by means of a crystalline undulator (CU) [6,35-38]. This device implies channeling in a periodically bent crystal. The use of a CU would allow to increase the radiation intensity and to decrease the radiation cone angle w.r.t. CR. However, the application of a CU is limited by channeling acceptance and dechanneling. Actually, the study of the radiation generation in a bent crystal is a necessary step for the development of a CU. In particular, one should study how the spectral intensity depends on the crystal alignment w.r.t. the beam, as well as on the crystal curvature. Additionally, one should develop and validate a simulation model that predicts the radiation spectra. 
An important factor for the realization of an innovative crystal-based radiation source is the choice of the crystalline material. The most frequently used material for channeling in bent crystals is silicon ( $\mathrm{Si}$ ), since the techniques for the production of high-quality bent Si monocrystals are well established and its lattice provides high electric field. However, even higher electric fields can be obtained in materials of higher Z, for instance germanium (Ge), which can be manufactured by similar procedures and with a similar quality as $\mathrm{Si}$ $[27,39,40]$. Therefore, higher- $Z$ crystals are very appealing to increase radiation intensity [41].

In this paper, we show the experimental results obtained via $\mathrm{CR}$ and VR radiation in ultrashort $\mathrm{Si}$ and Ge bent crystals at the Mainz Mikrotron (MAMI), using an $855 \mathrm{MeV}$ electron beam. We compared the experimental spectra with simulations as a function of curvature radius and crystal alignment, both for $\mathrm{Si}$ and $\mathrm{Ge}$ samples. In particular, the radiation in a Ge bent crystal and its dependence on the crystal curvature are studied for the first time.

\section{Experimental setup}

The experimental setup for both beam steering and radiation measurements at MAMI is shown in Fig. 1. In particular, the MAMI B $855 \mathrm{MeV}$ electron beam was used. Beam size and angular divergence in the plane of the crystal bending were $105 \mu \mathrm{m}$ and $21 \mu \mathrm{rad}$, respectively. The divergence was considerably less than the Lindhard angle, which is $232 \mu \mathrm{rad}$ for Si (111) and $274 \mu$ rad for Ge (111) for $855 \mathrm{MeV}$ electrons. The samples were $15 \mu \mathrm{m}$ thick, while their lateral size was optimized to suppress parasitic anticlastic deformation across the central region of the crystal, resulting in a cylindrical surface [42]. Small imperfections caused by the glueing procedure resulted in a residual variation of the (111) plane orientation [27]. The beam entered the samples in the center of their largest surfaces, i.e. traversing $15 \mu \mathrm{m}$ of material, as shown in the top-left corner of Fig. 1. The beam was aligned with the (111) planes, which were bent by the quasi-mosaic effect $[43,44]$.

Both crystals were mounted on a piezo-driven dynamical holder $[27,45]$, which allowed to modify the sample curvature $\left(\theta_{b}\right)$ without replacing the samples and without breaking the vacuum of the experimental apparatus. The holder was installed on a high-precision goniometer with 5 degrees of freedom. The goniometer was used to align the crystals with the electron beam. The measurements were done at three different bending angles of the (111) planes for the Si sample $(550,750$, and $1080 \mu \mathrm{rad})$ and three values of the (111) planes for the Ge sample (820, 1200, and $1430 \mu \mathrm{rad})$.

The electrons passed through the samples. Then, they were deflected by a dipole magnet onto a LYSO screen, which was used for the measurement of the deflection angle distribution.
The direction of the deflection of the beam was orthogonal to the deflection imposed by the magnet. The crystal alignment was carried out using the signal of a ionization chamber installed downstream of the samples. A more precise alignment was obtained via off-line analysis of the measured deflected beam distribution.

The measurements were carried out by considering different angular alignments between the samples and the beam $\left(\theta_{x}\right)$ :

- Channeling alignment, $\left|\theta_{x}\right| \ll \theta_{L}$;

- Volume reflection alignment, $-\theta_{b}<\theta_{x}<\theta_{L}$;

- Random alignment, $\theta_{x} \gg \theta_{L}$;

- "Anti volume reflection" (AVR) alignment, i.e. opposite to the VR one, $\theta_{L}<\theta_{x}<\theta_{b}$.

The emitted photons resulting from the interaction of the beam particles with the samples were collected by a $\mathrm{NaI}$ detector, in front of which a collimator allowed to accept only photons emitted within the $40 \mathrm{~mm}$ aperture positioned at $8.627 \mathrm{~m}$ from the crystal (see Fig. 1) [26,46,47].

\section{Experimental results}

For the sake of comparison with the spectra usually displayed in the literature, the spectral intensities are obtained by multiplying the experimental spectra collected by the NaI detector by the photon energy. Figure 2 shows the measured radiation spectral intensities for $\mathrm{Si}$ and $\mathrm{Ge}$ samples, for comparable sample curvatures. The bending angles were $750 \mu \mathrm{rad}$ for $\mathrm{Si}$ and $820 \mu \mathrm{rad}$ for Ge in Fig. 2a and $1080 \mu \mathrm{rad}$ for $\mathrm{Si}$ and $1200 \mu \mathrm{rad}$ for Ge in Fig. 2b. Each of these measurements was carried out in channeling alignment $\left(\theta_{x} \approx 0\right)$, in volume reflection alignment (at half of bending angle, i.e. $\theta_{x} \approx$ $\left.-\theta_{b} / 2\right)$, and at random alignment $\left(\theta_{x}>9 \mathrm{mrad}\right)$.

As expected, the intensity of radiation generated in the Ge bent sample was higher than that for $\mathrm{Si}$ at similar crystal-tobeam alignment due to the higher atomic number $Z$ of Ge. For instance, radiation at random alignment was enhanced for the Ge sample w.r.t. the Si sample roughly by a factor of 4. Nevertheless, one may notice that the $\mathrm{SiCR}$ is more intense than that for random oriented $\mathrm{Ge}$ in the $0.5-8 \mathrm{MeV}$ energy range thanks to the capability of channeling to enhance the radiation process. The $\mathrm{Si} C \mathrm{R}$ is also more peaked than the $\mathrm{Ge}$ $\mathrm{CR}$, which could be explained by considering a weaker contribution of multiple scattering to radiation [26] for a low- $Z$ material. On the other hand, both $\mathrm{CR}$ and VR radiation for the Ge sample were stronger than both $\mathrm{CR}$ and VR radiation for the $\mathrm{Si}$ one. Since this is indeed the first measurement of radiation enhancement in a bent Ge crystal, we rotated the crystal from random to channeling orientation, passing through VR, in order to have a better insight on radiation features. For bet- 


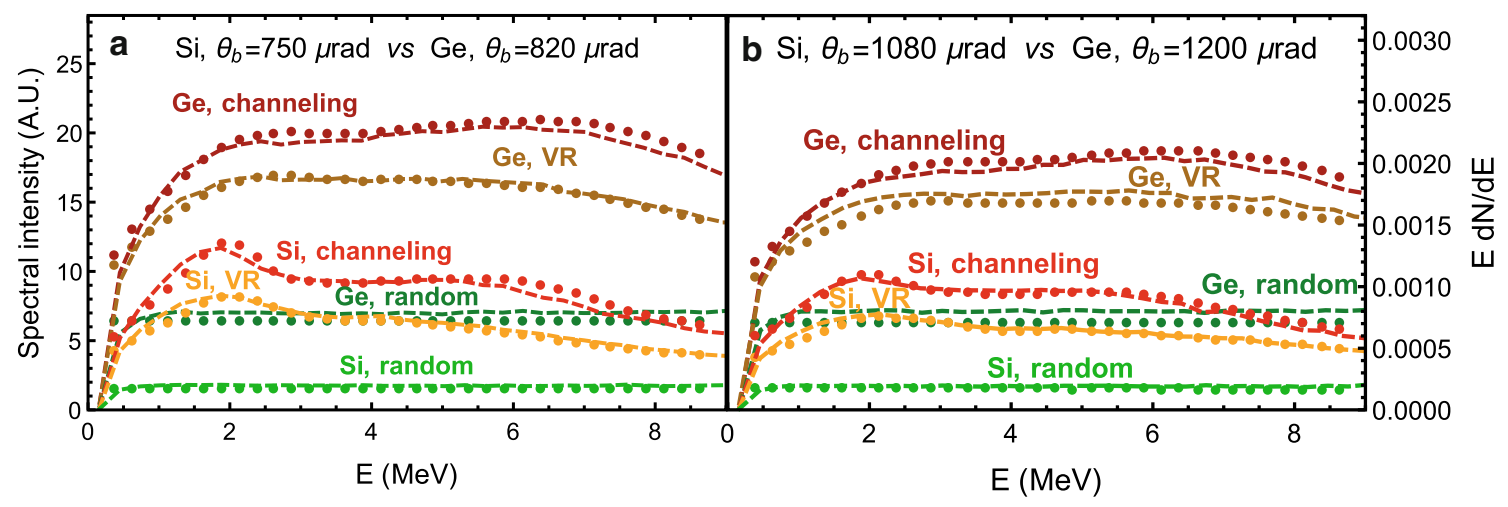

Fig. 2 Experimental (lines) and simulated (points) radiation spectral intensities for channeling $\left(\theta_{x} \sim 0 \mu \mathrm{rad}\right)$, volume reflection $\left(\theta_{x} \approx\right.$ $\left.-\theta_{b} / 2\right)$ and random orientation $\left(\theta_{x}>9 \mathrm{mrad}\right)$ for both $\mathrm{Si}$ and $\mathrm{Ge}$ samples. a The bending angles were $750 \mu \mathrm{rad}$ for $\mathrm{Si}$ and $820 \mu \mathrm{rad}$ for $\mathrm{Ge}$. b The bending angles were $1080 \mu \mathrm{rad}$ for $\mathrm{Si}$ and $1200 \mu \mathrm{rad}$ for $\mathrm{Ge}$
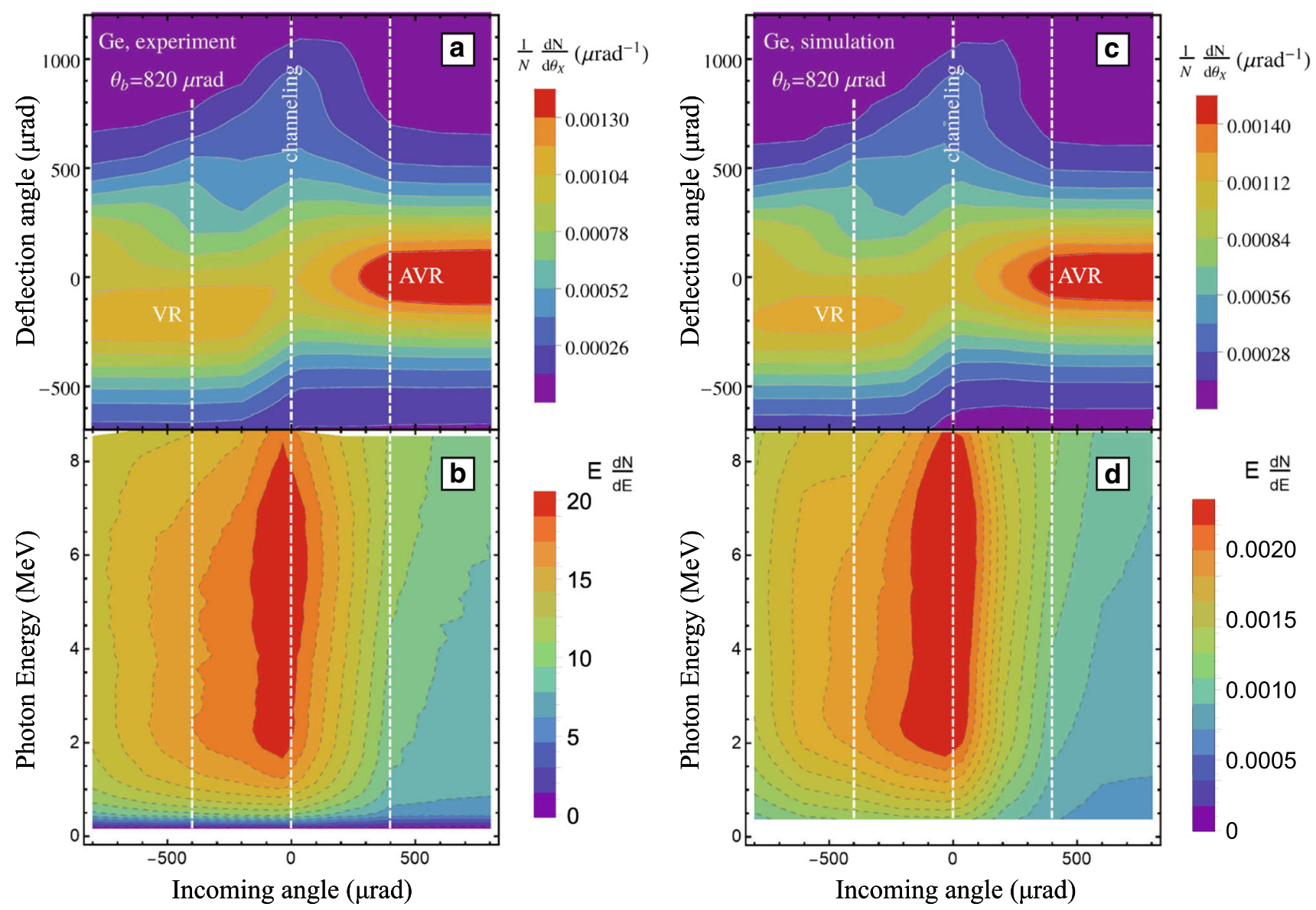

Fig. 3 Experimental (left) and simulated (right) deflection angle distributions (top) and radiation spectral intensities (bottom) with respect to the alignment of the Ge bent crystal with bending angle of $820 \mu \mathrm{rad}$

ter comprehensibility, we have also plotted the distributions of the deflected beam as a function of the crystal alignment, which was already published in [27]. These plots demonstrate that, as already shown for $\mathrm{Si}$ in [26], for a bent crystal the radiation intensity achievable in channeling condition is nearly maintained also for VR orientation (bottom plot of Fig. 3) in the whole angular acceptance $-\theta_{b}<\theta_{x}<\theta_{L}$.

To highlight this peculiar behavior of bent crystals, we compared the radiation spectral intensities for VR orientation $\left(\theta_{x} \approx-\theta_{b} / 2\right)$ and for the opposite case of AVR, for 


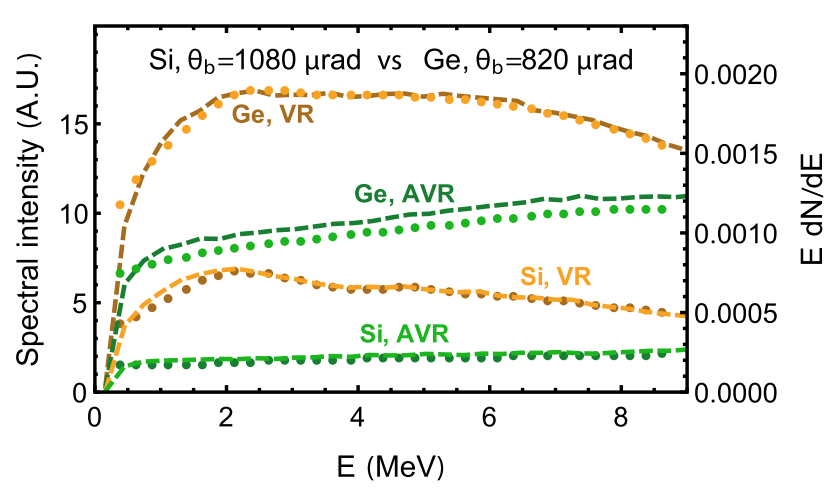

Fig. 4 Experimental (lines) and simulated (points) radiation spectral intensities for volume reflection $\left(-\theta_{b} / 2\right)$ and anti volume reflection orientation $\left(0<\theta_{x}<\theta_{b}\right)$ for both $\mathrm{Si}$ and $\mathrm{Ge}$ bent crystals. Bending angles are $1080 \mu \mathrm{rad}(\mathrm{Si})$ and $820 \mu \mathrm{rad}(\mathrm{Ge})$

which the crystal planes were misaligned w.r.t. the electron beam direction by an incoming angle $0<\theta_{x}<\theta_{b}$. As an example, in Fig. 4 the direct comparison of the VR and AVR spectra for the bending angles of $1080 \mu \mathrm{rad}$ and $820 \mu \mathrm{rad}$, for $\mathrm{Si}$ and Ge respectively, are presented. Here, a considerable enhancement of the radiation intensity when in VR alignment w.r.t. the case of an incoming angle in the opposite direction can be observed. Indeed, as is typical for straight crystals, the radiation intensity decreases quickly out of channeling condition, becoming harder while approaching to the coherent bremsstrahlung limit [47].

VR allows one to generate rather intense radiation, not far from $\mathrm{CR}$ one, without very strict requirements on the crystal-to-beam alignment, while the beam divergence could be larger than the Lindhard angle, unlike in the case of channeling. Since the VR angular acceptance is equal to the bending angle of the crystal, in principle one may suppose to increase the angular acceptance for intense gamma radiation generation by increasing the crystal bending angle as much as needed. For this reason, it is crucial to determine the dependence of the VR radiation spectrum on the crystal bending angle. Figure 5a, b display the channeling and VR spectral intensities for different crystal curvatures, respectively. $\mathrm{CR}$ was measured at three different values of bending angles $\theta_{b}$ for the Si crystal, namely 550, 750 and $1080 \mu \mathrm{rad}$ and three values for the Ge one, i.e. 820, 1200 and $1430 \mu \mathrm{rad}$. From Fig. 5a it is evident that the main trend for CR is the intensity decrease as the bending angle increases. This behaviour is due to the decrease of the fraction of channeling particles for larger curvature, for which dechanneling contribution is stronger, i.e., the dechanneling length is shorter, as demonstrated in [27]. This behavior is confirmed for both $\mathrm{Si}$ and $\mathrm{Ge}$ crystals. Figure $5 \mathrm{~b}$ confirms the same trend for the radiation intensity, decreasing as a function of the bending angle, also for VR. Indeed, the more the crystal is bent, the greater the contribution of $\gamma$ emitted by electrons crossing the crystal with trajectories more misaligned with respect to the crystal planes [24]. These photons are typically harder though radiation is less intense, resulting in a decrease of spectrum intensity. Furthermore, due to the decrease of the critical angle for stronger bending, less particles are volume captured in channeling condition and, even if captured, are dechanneled faster. Indeed, it has been demonstrated in [26] that the volume captured particles, i.e. the ones captured in channeling regime inside the crystal at the reflection point, are the main responsible for the maintenance of the CR peak also in VR orientation. Their decrease in number leads to a decrease of the VR radiation peak. For this reason, the radiation under VR orientation results in a mixture of different contributions coming from purely reflected particles and particles captured in channeling conditions. Nevertheless, one may notice that the intensity of VR radiation is comparable with the channeling case for all the values of bending radius considered for both $\mathrm{Si}$ and $\mathrm{Ge}$ bent crystals and the variation in its intensity depends slightly less on the curvature than in the case of CR.

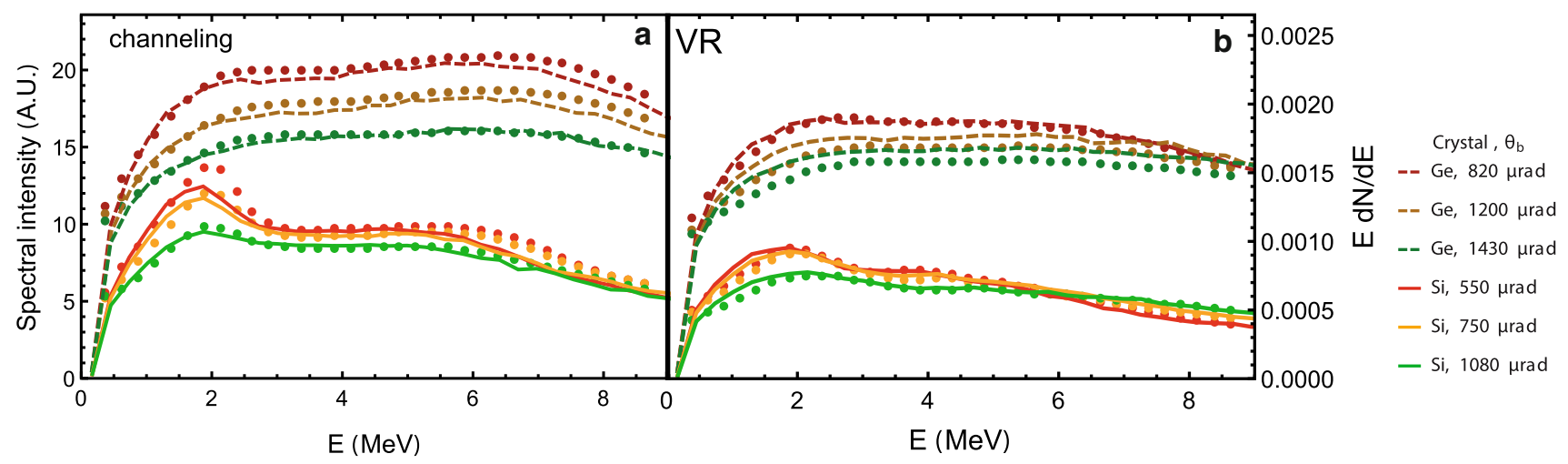

Fig. 5 Experimental (lines) and simulated (points) radiation spectral intensities for channeling (a) and volume reflection (b) alignments for both $\mathrm{Si}$ and Ge bent crystals with different values of bending angles. The
VR spectra were measured for the incoming angle $\theta_{x} \approx-\theta_{b} / 2$ except for the Si curvature $\theta_{b}=550 \mu \mathrm{rad}$, for which both the measurement and the simulation were taken at $\theta_{x} \approx-0.7 \theta_{b}$ 


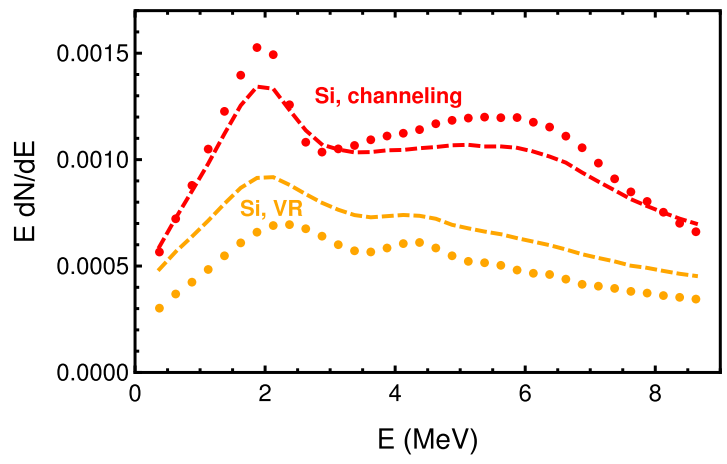

Fig. 6 Simulated radiation spectral intensities in case of channeling (red) and VR (yellow) orientations for the bent Si (left) and Ge (right) crystals, respectively. The bending angles were $750 \mu \mathrm{rad}$ for $\mathrm{Si}$ and

In conclusion, it can be stated that the overall angular acceptance for the production of high intense radiation in a bent crystal is approximately equal to the crystal bending angle, $\theta_{b}$, since $\mathrm{VR}$ radiation intensity is similar to that of the $\mathrm{CR}$ case. In addition, the radiation intensity decreases with $\theta_{b}$ increase. This fact is quite evident in Fig. 3, where it is shown that for the whole angular range from $\theta_{x}=0$ to $\theta_{x}=$ $-\theta_{b}=-820 \mu \mathrm{rad}$ the intensity of radiation is maintained close to the CR peak. One should pay by a small decrease of radiation intensity in order to provide considerably higher angular acceptance.

\section{Monte Carlo simulation and discussion}

The interaction of the charged particles of the electron beam with the bent crystals and the generation of radiation were simulated with the CRYSTALRAD code [48-50], which was already verified in several other experiments [23-28,32]. This code allows one to simulate classical charged particle trajectories, in straight and bent crystals, in the field of crystal planes or axes $[4,8]$ taking into account multiple and single Coulomb scattering on nuclei and electrons. The simulated spectral intensity $\mathrm{E}(\mathrm{dN} / \mathrm{dE})$ vs. $\mathrm{E}, \mathrm{dN} / \mathrm{dE}$ being the photon emission probability and $\mathrm{E}$ the photon energy, is calculated through the Baier-Katkov quasiclassical method [2]. The CRYSTALRAD code takes into account the crystal geometry, such as anticlastic curvature, crystal torsion, miscut angle (namely the angle between the physical surface and the atomic planes of the crystal), and geometrical size of the crystal.

First of all, all the spectra presented in Figs. 2, 3, 4 and 5 (dots) were well reproduced with the CRYSTALRAD simulation code. Some discrepancy in the radiation intensity between simulation outcomes and experimental results may be inferred to one of the following reasons or their combina-

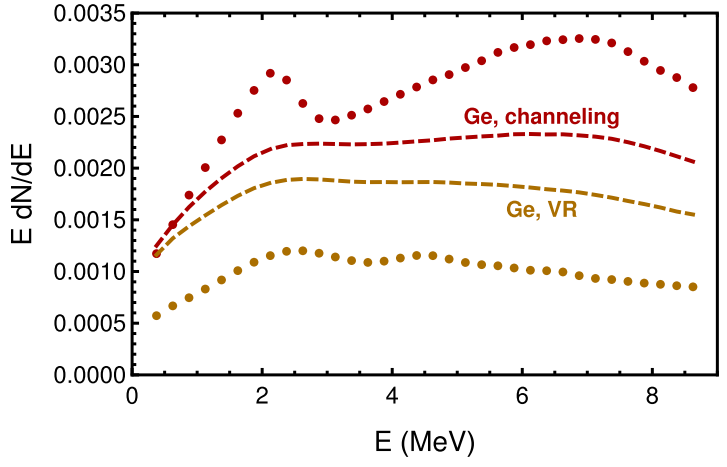

$820 \mu \mathrm{rad}$ for Ge as in Fig. 2a. The incoherent scattering is taken (not taken) into account in the dashed (dotted) line plots

tion: the chosen model of multiple and single Coulomb scattering, quantum corrections to Coulomb scattering at small angles [51,52], and the influence of high-index planes in case of "random orientation". Indeed, the weaker the scattering, the weaker the contribution of dechanneling of channeled and captured particles. At the same time, radiation in the case of random direction may be amplified by the presence of high-index skew planes, which may not have been perfectly avoided in choosing the random orientation.

In order to reveal the contribution of incoherent scattering, we recalculated the spectral intensities plotted in Fig. 2a for the case of the absence of incoherent scattering in the plane of crystal bending (see Fig. 6 dotted lines). By directly comparing the plots in Fig. 6 with incoherent scattering taken (dashed lines) and not taken (dotted lines) into account, it is clear that the contribution of incoherent scattering decreases the intensity of CR due to the dechanneling process, while increases the VR intensity through volume capture [26]. This trend is valid both for Si and Ge bent crystals, but it is more pronounced for the latter, being more affected by incoherent scattering due to its higher atomic number. In particular, in this latter case the channeling peak disappears when incoherent scattering is included, thereby highlighting the influence of dechanneling in the radiation spectrum.

The fairly good agreement between simulations and experiments allows us to exploit the Monte Carlo to investigate deeply the radiation generation in bent crystals. We focused our attention on the $\mathrm{Ge}$ case because its usage is a real novelty in the field and could open new possibilities for applications. In particular, we investigated deeply the dependence of $\mathrm{Ge}$ channeling spectra on curvature radius: Fig. 7 displays the radiation spectrum variation as a function of the ratio of the bending radius, $\mathrm{R}$, w.r.t. its critical value $R_{C}$, namely the radius at which the potential well depth disappears and channeling is not possible for value smaller than $R_{C}$. This picture confirms that the CR intensity increases with 


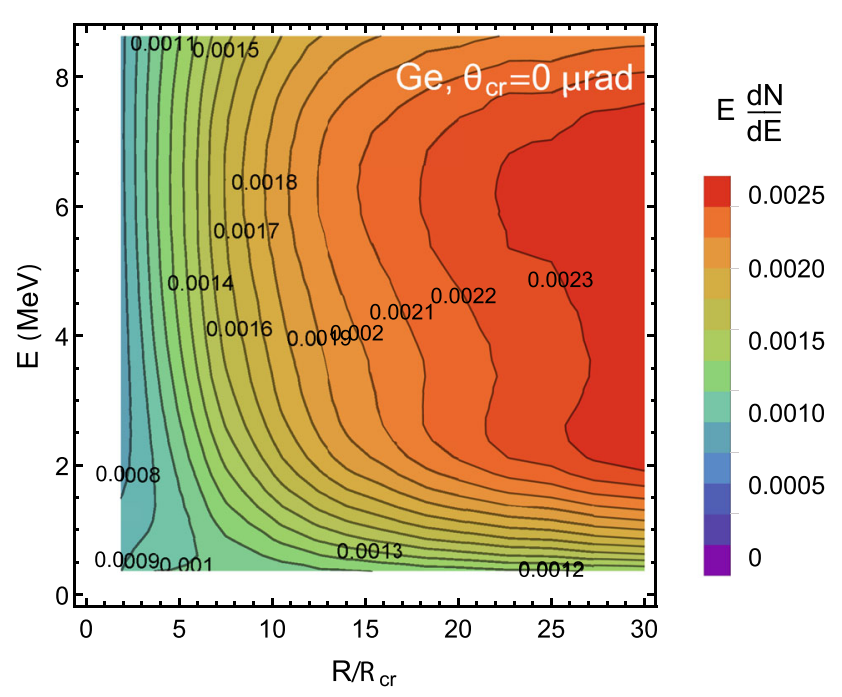

Fig. 7 Simulated radiation spectral intensities vs the ratio of the bending radius to its critical value for the Ge bent crystal

the increase of the bending radius, i.e. decrease of the bending angle, $\theta_{b}$. On the other hand, we have already shown in Figs. 2, 3 and 4 that also VR radiation decreases in intensity with $\theta_{b}$ increase, with a correspondent increase of the angular acceptance. In summary, one may find the best solution in terms of steering capability, radiation intensity, and angular acceptance for a particular application, such as intense radiation source or beam steerer, by tuning the bending angle of the crystal.

In general, crystals are very prospective radiation sources, since one can apply very intense electric fields naturally created. The channeling intense radiation can be exploited in photoproduction experiments or to investigate nuclear structures, as already possible for CB radiation [53].

With a bent crystal, one can also exploit the Volume Reflection radiation, which is not present among the interactions with straight crystals. This effect demonstrates comparable, albeit slightly lower, radiation intensity with the channeling case, while the angular acceptance is considerably larger and tunable by proper choice of the crystal bending angle. Thus, VR in a bent crystal may overcome the problem of low angular acceptance of CR in a straight crystal, providing comparable intensity of radiation. In particular, VR can be employed for applications in which intense radiation losses and beam steering can be combined.

\section{Conclusions}

An experiment to investigate the emitted radiation by $855 \mathrm{MeV}$ electrons in $15 \mu \mathrm{m}$ thick Si and Ge bent crystals has been carried out at the MAMI B line in Mainz (Germany). The radiation spectral intensities in case of planar channeling and volume reflection (VR) orientations have been measured and well reproduced by the CRYSTALRAD simulation code for three different values of the crystal curvature for the $\mathrm{Si}$ crystal as well as three values for the Ge one. Both experiment and simulation outcomes showed that the radiation intensity increases with the decrease of the bending angle, for both channeling (CR) and VR radiation cases.

The spectra for $\mathrm{Si}$ and Ge bent crystals with similar curvature were directly compared, demonstrating approximately a twofold increase in the radiation intensity for Ge in comparison with $\mathrm{Si}$. Nevertheless, the $\mathrm{Si} \mathrm{CR}$ is more peaked than the $\mathrm{Ge} C \mathrm{R}$, which could be explained by a weaker contribution of multiple scattering to radiation for a low-Z material. Finally, given its large angular acceptance and higher deflection efficiency than channeling, VR radiation can be exploited to produce intense radiation with poor emittance beams also using higher $\mathrm{Z}$ material than $\mathrm{Si}$ or $\mathrm{C}$, thereby opening the way for application in future accelerators if beam deflection combined with intense radiation generation is needed.

In summary, the results presented in this paper are relevant for the generation of $X$ - and $\gamma$-ray radiation in bent crystals, including higher- $Z$ crystalline materials and crystalline undulators [34,35]. Moreover, given the good agreement with experimental outcomes, the Monte Carlo model used in this paper may be exploited to predict the results for future experiments, which is also essential for the development of innovative radiation sources.

Acknowledgements We acknowledge partial support of the INFN through the CSN5 ELIOT and STORM experiments, the MC-INFN and the Grant73 OSCaR projects. We also acknowledge partial support by the European Commission through the N-LIGHT Project, GA 872196. M. Romagnoni acknowledges partial support from the ERC Consolidator Grant SELDOM G.A. 771642. We also acknowledge the CINECA award under the ISCRA initiative for the availability of high performance computing resources and support. We acknowledge Professor H. Backe for fruitful discussion and M. Rampazzo, A. Pitacco, and A. Minarello for technical assistance in dynamic holder realization.

Data Availability Statement This manuscript has no associated data or the data will not be deposited. [Authors' comment: All data generated or analysed during this study are included in this published article.]

Open Access This article is licensed under a Creative Commons Attribution 4.0 International License, which permits use, sharing, adaptation, distribution and reproduction in any medium or format, as long as you give appropriate credit to the original author(s) and the source, provide a link to the Creative Commons licence, and indicate if changes were made. The images or other third party material in this article are included in the article's Creative Commons licence, unless indicated otherwise in a credit line to the material. If material is not included in the article's Creative Commons licence and your intended use is not permitted by statutory regulation or exceeds the permitted use, you will need to obtain permission directly from the copyright holder. To view a copy of this licence, visit http://creativecomm ons.org/licenses/by/4.0/.

Funded by $\mathrm{SCOAP}^{3}$. 


\section{References}

1. M.L. Ter-Mikaelian, High-Energy Electromagnetic Processes in Condensed Media (Wiley, New York, 1972)

2. V.N. Baier, V.M. Katkov, V.M. Strakhovenko, Electromagnetic Processes at High Energies in Oriented Single Crystals (World Scientific, Singapore, 1998)

3. A.I. Akhiezer, N.F. Shul'ga, High-Energy Electrodynamics in Matter (Gordon \& Breach, New York, 1996)

4. Jens Lindhard, Influence of crystal lattice on motion of energetic charged particles. Mat. Fys. Medd. Dan. Vid. Selsk 34, 1-65 (1965)

5. M.A. Kumakhov, On the theory of electromagnetic radiation of charged particles in a crystal. Phys. Lett. A 57(1), 17-18 (1976)

6. V.G. Baryshevsky, A.O. Grubich, I.Ya. Dubovskaya, Generation of $\gamma$-quanta by channeled particles in the presence of a variable external field. Phys. Lett. A 77, 61-64 (1980)

7. V.V. Beloshitsky, M.A. Kumakhov, V.A. Muralev, Multiple scattering of channeling ions in crystals-II. Planar channeling. Radiat. Eff. 20, 95-109 (1973)

8. V.M. Biryukov, Y.A. Chesnekov, V.I. Kotov, Crystal Channeling and Its Applications at High-Energy Accelerators (Springer, 1996)

9. J.U. Andersen, E. Bonderup, R.H. Pantell, Channeling radiation. Annu. Rev. Nucl. Particle Sci. 33(1), 453-504 (1983)

10. A.H. Sørensen, E. Uggerhøj, Channelling and channelling radiation. Nature 325, 311-415 (1987)

11. R.A. Carrigan Jr., J. Ellison, Relativistic Channeling (Plenum Press, 1987)

12. V. Beloshitskii, S. Dabagov, Electromagnetic radiation at coherent and incoherent scattering of relativistic electrons in crystals. Sov. Phys. Tekh. Phys. 33, 939-942 (1988)

13. D. Myurrej, R.O. Avakyan, I.I. Miroshnichenko, T. Figut, Radiation emission by ultrarelativistic positrons moving in monocrystals near the crystallographic axes and ptanes. Zhurnal Ehksperimental'noj i Teoreticheskoj Fiziki 82, 1825-1832 (1982)

14. J. Bak et al., Channeling radiation from $2-55 \mathrm{GeV} / \mathrm{c}$ electrons and positrons: (i). Planar case. Nucl. Phys. B 254(0), 491-527 (1985)

15. N.A. Filatova, V.M. Golovatyuk, A.N. Iskakov, I.M. Ivanchenko, R.B. Kadyrov, N.N. Karpenko, T.S. Nigmanov, V.V. Palchik, V.D. Riabtsov, M.D. Shafranov, E.N. Tsyganov, I.A. Tyapkin, D.V. Uralski, A. Forycki, Z. Guzik, J. Wojtkowska, R.A. Carrigan, T.E. Toohig, C. Carmack, W.M. Gibson, I.J. Kim, C.R. Sun, M.D. Bavizhev, N.K. Bulgakov, N.I. Zimin, I.A. Grishaev, G.D. Kovalenko, B.I. Shramenko, E.I. Denisov, V.I. Glebov, V.V. Avdeichikov, Radiation from the channeling of $10-\mathrm{GeV}$ positrons by silicon single crystals. Phys. Rev. Lett. 48, 488-492 (1982)

16. R.A. Carrigan et al., Beam extraction studies at $900 \mathrm{GeV}$ using a channeling crystal. Phys. Rev. ST Accel. Beams 5, 043501 (2002)

17. W. Scandale, G. Arduini, M. Butcher, F. Cerutti, M. Garattini, S. Gilardoni, A. Lechner, R. Losito, A. Masi, D. Mirarchi, S. Montesano, S. Redaelli, R. Rossi, P. Schoofs, G. Smirnov, G. Valentino, D. Breton, L. Burmistrov, V. Chaumat, S. Dubos, J. Maalmi, V. Puill, A. Stocchi, E. Bagli, L. Bandiera, G. Germogli, V. Guidi, A. Mazzolari, S. Dabagov, F. Murtas, F. Addesa, G. Cavoto, F. Iacoangeli, L. Ludovici, R. Santacesaria, P. Valente, F. Galluccio, A.G. Afonin, Y. Chesnokov, A.A. Durum, V.A. Maisheev, Y. Sandomirskiy, A.A. Yanovich, A.D. Kovalenko, A.M. Taratin, A.S. Denisov, Y. Gavrikov, Y. Ivanov, L.P. Lapina, L.G. Malyarenko, V.V. Skorobogatov, T. James, G. Hall, M. Pesaresi, M. Raymond, Observation of channeling for $6500 \mathrm{GeV} / \mathrm{c}$ protons in the crystal assisted collimation setup for LHC. Phys. Lett. B 758, 129-133 (2016)

18. D. Lietti et al., Radiation emission phenomena in bent silicon crystals: theoretical and experimental studies with $120 \mathrm{GeV} / \mathrm{c}$ positrons. Nucl. Instrum. Methods Phys. Res. B 283, 84-92 (2012)
19. A.G. Afonin, V.T. Baranov, G .I. Britvich, A .P. Bugorskii, V .I. Kotov, A .E. Kushnirenko, V .A. Maisheev, V .A. Pikalov, V .N. Chepegin, Yu A Chesnokov, I .A. Yazynin, Yu M. Ivanov, V .V. Skorobogatov, Investigation of the emission of photons induced in the volume reflection of $10-\mathrm{GeV}$ positrons in a bent silicon single crystal. JETP Lett. 88, 414-417 (2008)

20. W. Scandale, G. Arduini, M. Butcher, F. Cerutti, S. Gilardoni, L. Lari, A. Lechner, R. Losito, A. Masi, A. Mereghetti, E. Metral, D. Mirarchi, S. Montesano, S. Redaelli, P. Schoofs, G. Smirnov, E. Bagli, L. Bandiera, S. Baricordi, P. Dalpiaz, V. Guidi, A. Mazzolari, D. Vincenzi, G. Claps, S. Dabagov, D. Hampai, F. Murtas, G. Cavoto, M. Garattini, F. Iacoangeli, L. Ludovici, R. Santacesaria, P. Valente, F. Galluccio, A.G. Afonin, M.K. Bulgakov, YuA Chesnokov, V.A. Maisheev, I.A. Yazynin, A.D. Kovalenko, A.M. Taratin, V.V. Uzhinskiy, YuA Gavrikov, YuM Ivanov, L.P. Lapina, W. Ferguson, J. Fulcher, G. Hall, M. Pesaresi, M. Raymond, V. Previtali, Optimization of the crystal assisted collimation of the SPS beam. Phys. Lett. B 726(1-3), 182-186 (2013)

21. W. Scandale et al., Experimental study of the radiation emitted by $180 \mathrm{GeV} / \mathrm{c}$ electrons and positrons volume-reflected in a bent crystal. Phys. Rev. A 79, 012903 (2009)

22. U. Wienands, T.W. Markiewicz, J. Nelson, R.J. Noble, J.L. Turner, U.I. Uggerhøj, T.N. Wistisen, E. Bagli, L. Bandiera, G. Germogli, V. Guidi, A. Mazzolari, R. Holtzapple, M. Miller, Observation of deflection of a beam of multi-GeV electrons by a thin crystal. Phys. Rev. Lett. 114, 074801 (2015)

23. T.N. Wistisen, R.E. Mikkelsen, U.I. Uggerhøj, U. Wienands, T.W. Markiewicz, S. Gessner, M.J. Hogan, R.J. Noble, R. Holtzapple, S. Tucker, V. Guidi, A. Mazzolari, E. Bagli, L. Bandiera, A. Sytov, (SLAC E-212 Collaboration), Observation of quasichanneling oscillations. Phys. Rev. Lett. 119, 024801 (2017)

24. L. Bandiera et al., On the radiation accompanying volume reflection. Nucl. Instrum. Methods Phys. Res. Sect. B 309, 135-140 (2013)

25. L. Bandiera et al., Broad and intense radiation accompanying multiple volume reflection of ultrarelativistic electrons in a bent crystal. Phys. Rev. Lett. 111, 255502 (2013)

26. L. Bandiera et al., Investigation of the electromagnetic radiation emitted by sub-GeV electrons in a bent crystal. Phys. Rev. Lett. 115, 025504 (2015)

27. A.I. Sytov, L. Bandiera, D. De Salvador, A. Mazzolari, E. Bagli, A. Berra, S. Carturan, C. Durighello, G. Germogli, V. Guidi, P. Klag, W. Lauth, G. Maggioni, M. Prest, M. Romagnoni, V.V. Tikhomirov, E. Vallazza, Steering of sub-GeV electrons by ultrashort $\mathrm{Si}$ and $\mathrm{Ge}$ bent crystals. Eur. Phys. J. C 77, 901 (2017)

28. A. Mazzolari et al., Steering of a sub-GeV electron beam through planar channeling enhanced by rechanneling. Phys. Rev. Lett. 112, 135503 (2014)

29. C.F. Nielsen, U.I. Uggerhøj, R. Holtzapple, T.W. Markiewicz, B.C. Benson, E. Bagli, L. Bandiera, V. Guidi, A. Mazzolari, U. Wienands, Photon emission by volume reflected electrons in bent crystals. Phys. Rev. Accel. Beams 22, 114701 (2019)

30. A.M. Taratin, S.A. Vorobiev, Volume reflection of high-energy charged particles in quasi-channeling states in bent crystals. Phys. Lett. A 119, 425-428 (1987)

31. YuA Chesnokov, V.I. Kotov, V.A. Maisheev, I.A. Yazynin, Radiation of photons in process of charged particle volume reflection in bent monocrystal. J. Instrum. 3(02), P02005-P02005 (2008)

32. V. Guidi, L. Bandiera, V.V. Tikhomirov, Radiation generated by single and multiple volume reflection of ultrarelativistic electrons and positrons in bent crystals. Phys. Rev. A 86, 042903 (2012)

33. Andrei Seryi, Future prospects of accelerator science for particle physics. Nucl. Instrum. Methods Phys. Res. Sect. A 623(1), 23-28 (2010)

34. U. Wienands, S. Gessner, M.J. Hogan, T.W. Markiewicz, T. Smith, J. Sheppard, U.I. Uggerhøj, J.L. Hansen, T.N. Wistisen, E. Bagli, 
L. Bandiera, G. Germogli, A. Mazzolari, V. Guidi, A. Sytov, R.L. Holtzapple, K. McArdle, S. Tucker, B. Benson, Channeling and radiation experiments at SLAC. Nucl. Instrum. Methods Phys. Res. Sect. B: Beam Interact. Mater. Atoms 402, 11-15 (2017). Proceedings of the 7th International Conference Channeling 2016: Charged \& Neutral Particles Channeling Phenomena

35. A.V. Korol, A.V. Solov'yov, W. Greiner, Channeling and radiation in periodically bent crystals, vol. 69 (Springer, 2013)

36. E. Bagli, L. Bandiera, V. Bellucci, A. Berra, R. Camattari, D. De Salvador, G. Germogli, V. Guidi, L. Lanzoni, D. Lietti, A. Mazzolari, M. Prest, V.V. Tikhomirov, E. Vallazza, Experimental evidence of planar channeling in a periodically bent crystal. Eur. Phys. J. C 74(10), 3114-1-3114-7 (2014)

37. R. Camattari, L. Bandiera, V. Tikhomirov, M. Romagnoni, E. Bagli, G. Germogli, A. Sytov, T. Maiolino, M. Tamisari, A. Mazzolari, V. Guidi, G. Cavoto, Silicon crystalline undulator prototypes: manufacturing and x-ray characterization. Phys. Rev. Accel. Beams 22, 044701 (2019)

38. V.G. Baryshevsky, V.V. Tikhomirov, Crystal undulators: from the prediction to the mature simulations. Nucl. Instrum. Methods Phys. Res. B 309, 30-36 (2013)

39. D. De Salvador, E. Bagli, O. Lytovchenko, A. Mazzolari, S. Carturan, G. Della Mea, V. Guidi, M. Bazzan, N. Argiolas, A. Carnera, D. Bolognini, S. Hasan, M. Prest, E. Vallazza, Steering of an ultrarelativistic proton beam by a bent germanium crystal. Appl. Phys. Lett. 98, 234102 (2011)

40. D. De Salvador, G. Maggioni, S. Carturan, M. Bazzan, N. Argiolas, A. Carnera, M. Dalla Palma, G. Della Mea, E. Bagli, A. Mazzolari, L. Bandiera, V. Guidi, D. Lietti, A. Berra, G. Guffanti, M. Prest, E. Vallazza, Highly bent (110) ge crystals for efficient steering of ultrarelativistic beams. J. Appl. Phys. 114,154902 (2013)

41. H. Shen, Q. Zhao, F.S. Zhang, Gennady B Sushko, Andrei V Korol, Andrey V Solov'yov, Channeling and radiation of $855-\mathrm{MeV}$ electrons and positrons in straight and bent tungsten (110) crystals. Nucl. Instrum. Methods Phys. Res. B 424, 26-36 (2018)

42. G. Germogli, A. Mazzolari, L. Bandiera, E. Bagli, V. Guidi, Manufacturing and characterization of bent silicon crystals for studies of coherent interactions with negatively charged particles beams. Nucl. Instrum. Methods Phys. Res. B 355, 81-85 (2015)
43. Yu. Ivanov, A. Petrunin, V. Skorobogatov, Observation of the elastic quasi-mosaicity effect in bent silicon single crystals. JETP Lett. 81, 99-101 (2005)

44. R. Camattari, V. Guidi, V. Bellucci, A. Mazzolari, The 'quasimosaic' effect in crystals and its applications in modern physics. J. Appl. Cryst. 48, 977-989 (2015)

45. D. De Salvador, S. Carturan, A. Mazzolari, E. Bagli, L. Bandiera, C. Durighello, G. Germogli, V. Guidi, P. Klag, W. Lauth, G. Maggioni, M. Romagnoni, A. Sytov, Innovative remotely-controlled bending device for thin silicon and germanium crystals. J. Instrum. 13(04), C04006-C04006 (2018)

46. D. Lietti et al., Radiation emission phenomena in bent silicon crystals: theoretical and experimental studies with $120 \mathrm{GeV} / \mathrm{c}$ positrons. Nucl. Instrum. Methods Phys. Res. Sect. B 283, 84-92 (2012)

47. H. Backe, P. Kunz, W. Lauth, A. Rueda, Planar channeling experiments with electrons at the $855 \mathrm{MeV}$ Mainz Microtron MAMI. Nucl. Instrum. Methods Phys. Res. B 266, 3835-3851 (2008)

48. A.I. Sytov, V.V. Tikhomirov, L. Bandiera, Simulation code for modeling of coherent effects of radiation generation in oriented crystals. Phys. Rev. Accel. Beams 22, 064601 (2019)

49. L. Bandiera, E. Bagli, V. Guidi, V.V. Tikhomirov, RADCHARM++: a $\mathrm{C}++$ routine to compute the electromagnetic radiation generated by relativistic charged particles in crystals and complex structures. Nucl. Instrum. Methods Phys. Res. A 355, 44-48 (2015)

50. A.I. Sytov, V.V. Tikhomirov, CRYSTAL simulation code and modeling of coherent effects in a bent crystal at the LHC. Nucl. Instrum. Methods Phys. Res. B 355, 383-386 (2015)

51. Victor V. Tikhomirov, Quantum features of high energy particle incoherent scattering in crystals. Phys. Rev. Accel. Beams 22, 054501 (2019)

52. V.V. Tikhomirov, Erratum: Quantum features of high energy particle incoherent scattering in crystals [Phys. Rev. Accel. Beams 22, 054501 (2019)]. Phys. Rev. Accel. Beams 23, 039901 (2020)

53. C.A. Paterson et al., Photoproduction of $\Lambda$ and $\Sigma^{0}$ hyperons using linearly polarized photons. Phys. Rev. C 93, 065201 (2016) 